\title{
Integrated silicon optofluidic ring resonator
}

\author{
Genni Testa, ${ }^{1}$ Yujian Huang, ${ }^{2}$ Pasqualina M. Sarro, ${ }^{2}$ Luigi Zeni, ${ }^{3}$ and Romeo Bernini $\left.{ }^{1}, a\right)$ \\ ${ }^{1}$ IREA-CNR, Via Diocleziano 328, 80124 Napoli, Italy \\ ${ }^{2}$ ECTM-DIMES, TU Delft, NL-2600 GB Delft, The Netherlands \\ ${ }^{3}$ DII, Seconda Università di Napoli, Via Roma 29, 81031 Aversa, Italy
}

(Received 27 July 2010; accepted 11 September 2010; published online 1 October 2010)

\begin{abstract}
The feasibility of an integrated silicon optofluidic ring resonator is demonstrated. Liquid core antiresonant reflecting optical waveguides are used to realize a rectangular ring resonator with a multimode interference liquid core coupler between the ring and the bus waveguide. In this configuration the same waveguide used to confine the light is able to deliver the liquid sample without the need for any additional microfluidics, resulting in a very compact device with a required liquid volume of about $0.11 \mathrm{nl}$. Optical characterization shows that ring with a good quality factor $(\mathrm{Q} \sim 800)$ can be achieved in good agreement with the numerical results. () 2010 American Institute of Physics. [doi:10.1063/1.3496027]
\end{abstract}

Optical ring resonators have been used as high sensitivity miniaturized biological or biochemical sensors. Several devices geometries as microspheres, ${ }^{1}$ microrings, ${ }^{2}$ and microdisks ${ }^{3}$ have been investigated. The high sensitivity of these devices relies on the interaction length between the light and sample that is not limited by the physical length but is related to the number of revolutions of light in the resonator, characterized by the quality factor Q. Basically, all the resonator-based sensors proposed are based on following two major sensing mechanisms: refractive index sensing ${ }^{1-3}$ and absorption sensing. ${ }^{4}$ In order to achieve high performance these structures require an optimized integration of the optical and microfluidic parts. In many cases, the design of optical part has been privileged respect to microfluidic one sacrificing the feasibility and the multiplexing capability of the device. Recently, a great effort has been devoted to find new integration strategies like liquid core ring resonator ${ }^{5}$ or to realize effective fluidics in high sensitivity integrated ring resonator. ${ }^{6}$ However, until now a fully integrated ring resonator has not yet been proposed. In this paper, we demonstrate the feasibility of an integrated silicon optofluidic ring resonator. The device is based on liquid core antiresonant reflecting optical waveguides (ARROWs). Light propagation in these waveguides occurs within the inner liquid-filled core, thanks to a surrounding multilayer stack designed to operate as a high reflectivity Fabry-Perot mirror. ${ }^{7,8}$ Using this approach, the waveguide used to realize the ring resonator is also used for delivering the sample under analysis; this results in a strong light-liquid coupling regime that makes the ARROW waveguides very attractive for sensing application. However, because of ARROWs are leaky waveguides, an accurate design is necessary in order to minimize the optical losses and achieve good performances.

The layout of the proposed device is shown in Fig. 1. Four $90^{\circ}$-bent ARROW waveguides are used to form a rectangular ring resonator and a multimode interference (MMI) liquid core ARROW coupler with a 50:50 splitting ratio acts as coupling element between the ring and the bus waveguide.

In comparison to conventional arch-shape waveguide, the use of $90^{\circ}$-bent ARROW waveguides permits to strongly

${ }^{a)}$ Electronic mail: bernini.r@irea.cnr.it. reduce the bending losses ${ }^{9,10}$ improving the ring quality factor. The rectangular waveguide's core has a width $d_{c}$ $=2 \mu \mathrm{m}$ and a height of $10 \mu \mathrm{m}$ in order to support a TEpolarized optical mode with respect to the lateral confinement. In fact, since in an ARROW waveguide the guidance mechanism relies on the Fresnel reflection from multiple cladding layers, different light polarization states undergo different propagation losses. In particular, the losses for the TE-polarized light result significantly lower than that for a TM-polarized light, especially at high incidence angle, as it happens in the bent region. ${ }^{9}$

The width $\mathrm{W}_{\mathrm{MMI}}=4 \mu \mathrm{m}$ and the length $\mathrm{L}_{\mathrm{MMI}}$ $=2 \mathrm{n}_{\mathrm{c}} \mathrm{W}_{\text {MMI }}^{2} / \lambda=67.17 \mu \mathrm{m}$ of the MMI ARROW coupler were properly chosen for a balanced splitting ratio, at the working wavelength of $\lambda=635 \mathrm{~nm}$ and for a water-filled liquid core $\left(\mathrm{n}_{\mathrm{c}}=1.33\right)$. This results in a rectangular ring resonator with a total round-trip length $\mathrm{L}_{\mathrm{RT}}=174.4 \mu \mathrm{m}$. In order to provide a high coupling efficiency with single mode optical fibers, linear tapered waveguides were used to connect the input and output ports of the MMI coupler with the input and output straight access waveguides of $5 \mu \mathrm{m}$ width.

Liquid core ARROW waveguides were realized to enable low-loss single mode propagation at the wavelength of $\lambda=635 \mathrm{~nm}$ and for a core refractive index of $\mathrm{n}_{\mathrm{c}}=1.33$. The waveguide channels were defined by silicon dry etching followed by the realization of the antiresonant cladding layers by depositing a silicon dioxide $\left(\mathrm{n}_{2}=1.46\right)$ layer by low pressure plasma-enhanced chemical vapor deposition, and a titanium oxide $\left(\mathrm{n}_{1}=2.49\right)$ layer by atomic layer deposition (ALD). Further details of the fabrication process are reported elsewhere. ${ }^{8}$ The resulting layout provides a very compact device with a total length of only $2.5 \mathrm{~mm}$ and with a required liquid volume of about $0.11 \mathrm{nl}$. A scanning electron micro-

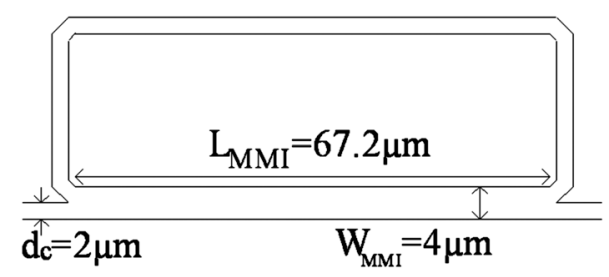

FIG. 1. Schematic geometry of the ring resonator. 


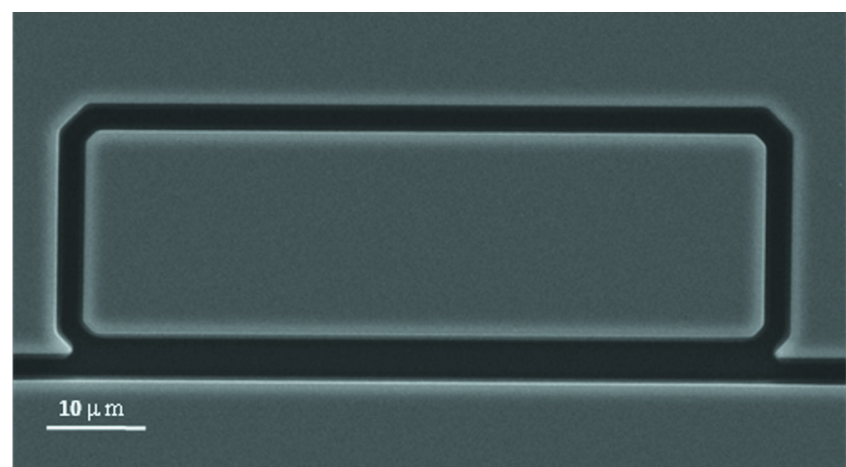

FIG. 2. (Color online) SEM picture of the fabricated device.

scope (SEM) image of the device is depicted in Fig. 2. The image illustrates the bottom wafer before adding the upper wafer on top of it. ${ }^{8,10}$

Two-dimensional (2D) finite-difference time-domain method (OmniSim,@Photon Design) was used to analyze the spectral response of the ring resonator. The input mode profile was calculated with a $2 \mathrm{D}$ mode solver (FIMMWAVE, ${ }^{\circ}$ Photon Design). In particular, the fundamental TE-mode of a $2 \mu \mathrm{m}$ wide ARROW waveguide was assumed as input field; it was modeled as a light pulse centered at $\lambda \sim 640 \mathrm{~nm}$ with a full width at half maximum (FWHM) linewidth of $\Delta \lambda_{\text {FWHM }} \sim 5 \mathrm{~nm}$. The refractive index of the core was set to $\mathrm{n}_{\mathrm{c}}=1.32$ (methanol) and perfectly matched layer (PML) boundary conditions were used at the edge of the computational domain. In order to evaluate the influence on the splitting ratio of a slight different core refractive index, we first have simulated the MMI coupler by considering $\lambda$ $=640 \mathrm{~nm}$ and $\mathrm{n}_{\mathrm{c}}=1.32$, obtaining a 49.6:50.4 splitting ratio between the two output ports of the MMI coupler.

Figure 3 shows the simulation results. The spectral information has been derived by performing a discrete Fourier transform of the time domain data. Resonances can be observed in the normalized transmitted spectrum at the output of the bus waveguide. In fact, due to constructive interference in the resonator, light couples into the ring and causes the transmission to drop at a well defined wavelengths. The resulting free spectral range (FSR) evaluated from the simu-

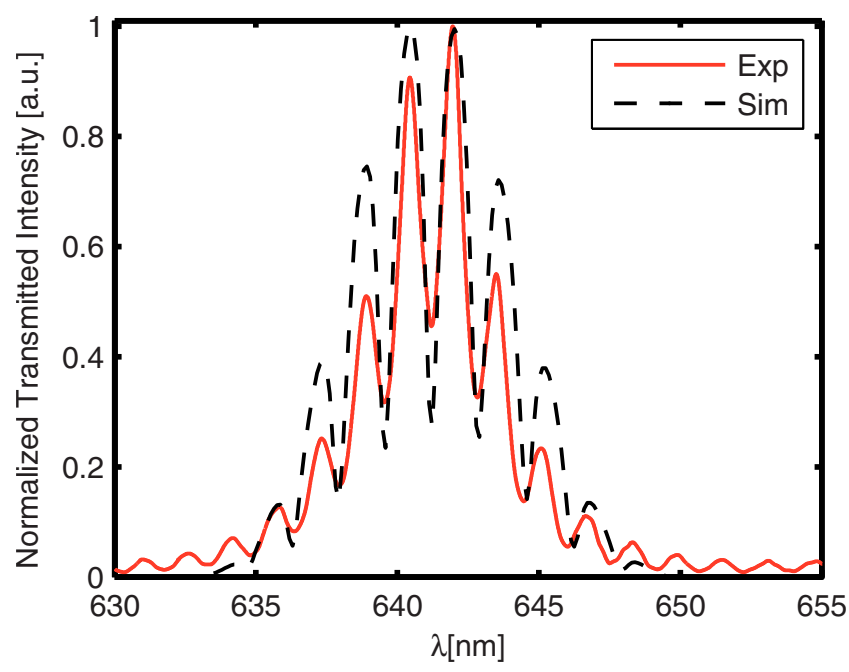

FIG. 3. (Color online) Experimental (solid lines) and simulated (dashed lines) normalized transmitted intensity for methanol filled ring resonator $\left(\mathrm{n}_{\mathrm{c}}=1.32\right)$.

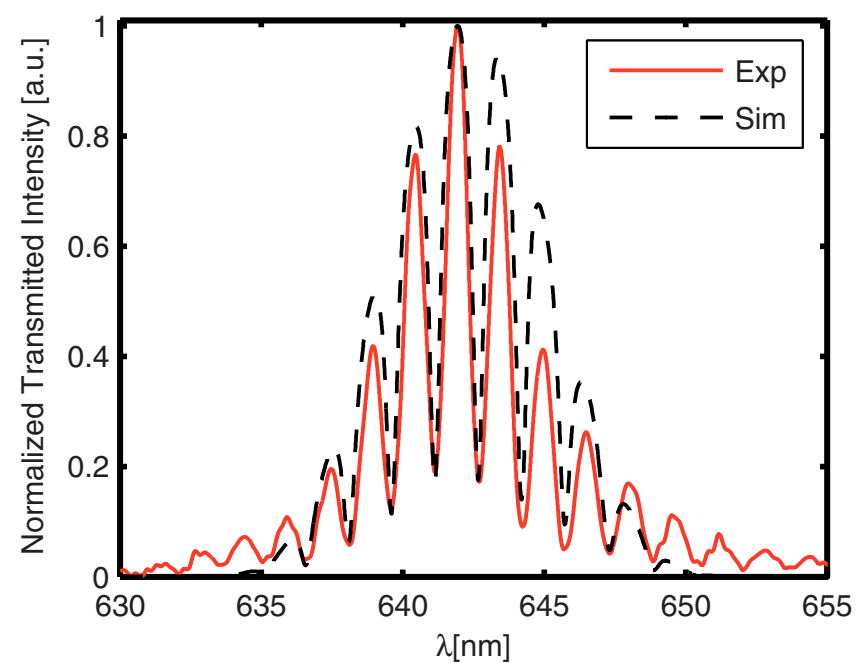

FIG. 4. (Color online) Experimental (solid lines) and simulated (dashed lines) normalized transmitted intensity for DMF filled ring resonator $\left(\mathrm{n}_{\mathrm{c}}=1.43\right)$.

lated spectrum is $\mathrm{FSR}_{\mathrm{s}} \sim 1.65 \mathrm{~nm}$ around $\lambda=640 \mathrm{~nm}$.

For the optical measurements, light from a diode laser $(\lambda=640 \mathrm{~nm}, \mathrm{FWHM}=5 \mathrm{~nm})$ was end-coupled via a single mode optical fiber (4.3 $\mu \mathrm{m}$ core diameter) into the liquid core bus waveguide. The transmitted light were collected by another single mode optical fiber and sent to a high resolution spectrometer. For an optimized coupling efficiency, an accurate optical alignment between the bus waveguide core and optical fiber has been achieved by employing a micrometer translation stage. Optical measurements were performed by filling the entire device through the open-ended hollow core bus waveguide with methanol $\left(\mathrm{n}_{\mathrm{c}}=1.32\right)$ by capillary action. The measured total loss of the device is $\sim 22 \mathrm{~dB}$.

In Fig. 3 the measured normalized transmitted spectrum from the device is shown. From measurements a $\mathrm{FSR}_{\mathrm{s}}$ $\sim 1.62 \mathrm{~nm}$ around $\lambda=640 \mathrm{~nm}$ and an extinction ratio (ER) of $3.42 \mathrm{~dB}$ were estimated. For comparison, in Fig. 3 the simulated spectrum is also reported. As can be seen, there is a good agreement between experimental and numerical results, which give an extinction ratio of $\mathrm{ER}_{\mathrm{s}} \sim 5.74 \mathrm{~dB}$.

The measured resonance linewidth (FWHM) is $0.88 \mathrm{~nm}$, leading to a quality factor $\mathrm{Q} \sim 740$, while from simulation it results $\mathrm{Q}_{\mathrm{s}} \sim 1150$. This difference is mainly due to the $2 \mathrm{D}$ geometry of the simulated device that does not take into account for modal attenuation of ARROW waveguide due to the vertical confinement. For this reason from simulations it results a reduced round-trip ring losses and a larger quality factor $\mathrm{Q}$ with respect to the experimental results.

In an ARROW waveguide the propagation losses can be made lower by employing a higher refractive index fluid filling the core. ${ }^{11}$ This also slightly reduces the bending losses, giving rise to an overall improving of the performances of the resonator. For this reason we have repeated the optical characterization of the device by filling the hollow core with the dimethylformamide (DMF), having a refractive index $n_{c}=1.43$. The measured transmitted spectrum is depicted in Fig. 4. As expected, there is an improvement in terms of ER, which became ER $7.64 \mathrm{~dB}$, and also the quality factor $\mathrm{Q} \sim 821$ results slightly increased. Moreover, since a higher refractive index is used, a reduced free spec- 
tral range $\mathrm{FSR}_{\mathrm{s}} \sim 1.55 \mathrm{~nm}$ results from the interference effect in the resonator. In Fig. 4, the simulated spectrum obtained by modeling the device with $\mathrm{n}_{\mathrm{c}}=1.43$ is also shown. From the spectrum we have estimated $\mathrm{ER}_{\mathrm{s}} \sim 7.65 \mathrm{~dB}$, $\mathrm{FSR}_{\mathrm{s}} \sim 1.53 \mathrm{~nm}$, and $\mathrm{Q}_{\mathrm{s}} \sim 1380$. These results are in agreement with the experimental ones and confirm that by reducing the attenuation losses due to the ARROW confinement, an improvement of the ring performances can be obtained. However, in order to get higher $\mathrm{Q}$ factor, further improvement of the round-trip losses with respect to the DMF-filled core ring configuration is required. Thanks to the wellknown ARROW properties, this can be obtained if only one more bilayers is added during fabrication procedure to the multilayer stack composing the Fabry-Perot mirror; in this case the attenuation losses of the waveguides are reduced by a factor of $7 .{ }^{11}$

Finally, we have evaluated the bulk refractive index sensitivity. The wavelength shift was deduced using the following relation: $\Delta \lambda=\lambda \Delta \mathrm{n} / \mathrm{n}$, where $\Delta \mathrm{n} / \mathrm{n}$ was deduced from the free-spectral range measurements. The obtained sensitivity is approximately $260 \mathrm{~nm} / \mathrm{RIU}$. This value is comparable with the theoretical sensitivity of $480 \mathrm{~nm} / \mathrm{RIU}$ obtained by the simulations. This high value can be explained by considering that the refractive index sensitivity is governed by the achievable partial sensitivity $S_{w}=\partial n_{\text {eff }} / \partial n_{c}$ that takes into account of the optical mode fraction that interacts with the sample. In a liquid core ARROW waveguide the mode field is almost completely confined into the liquid core of the waveguide $\left(S_{\mathrm{w}} \approx 1\right)$, so the interaction of the sample and field is strongly enhanced as compared to solid core waveguides in which only the evanescent part of the field is sensitive to the surrounding liquid medium.

As concerning the surface sensing by numerical simulation we have estimated a shift $\Delta \lambda / \lambda$ of about $2 \mathrm{e}-8$ for a $1 \mathrm{~nm}$ thick layer $(\mathrm{n}=1.45)$. This value is quite low compared to conventional solid core resonator, ${ }^{12}$ and can be explained taking into account that, in an ARROW waveguide, the mode field intensity at the core-cladding interface is very low and only a very small fraction of the optical mode interacts with the sample.

In conclusion, we have demonstrated the feasibility of an integrated optofluidic ring resonator. In the proposed device the optical part, used to realize the ring, and the microfluidic part, used to deliver the sample, are integrated together. This configuration avoids the need for any additional microfluidics, resulting in a very compact device with a required liquid volume of about $0.11 \mathrm{nl}$. The numerical simulations and the experimental results show that ring resonator with good quality factor can be achieved with interesting application especially in the sensing field.

The authors thank the IC Process Group of DIMES for help in device fabrication.

${ }^{1}$ F. Vollmer, D. Braun, A. Libchaber, M. Khoshsima, I. Teraoka, and S Arnold, Appl. Phys. Lett. 80, 4057 (2002).

${ }^{2}$ C. Chao and L. Guo, Appl. Phys. Lett. 83, 1527 (2003).

${ }^{3}$ E. Krioukov, D. J. W. Klunder, A. Driessen, J. Greve, and C. Otto, Opt Lett. 27, 1504 (2002)

${ }^{4}$ A. Nitkowski, L. Chen, and M. Lipson, Opt. Express 16, 11930 (2008).

${ }^{5}$ I. M. White, H. Oveys, and X. Fan, Opt. Lett. 31, 1319 (2006).

${ }^{6}$ C. F. Carlborg, K. B. Gylfason, A. Kaźmierczak, F. Dortu, M. J. Bañuls Polo, A. Maquieira Catala, G. M. Kresbach, H. Sohlström, T. Moh, L. Vivien, J. Popplewell, G. Ronan, C. A. Barrios, G. Stemme, and W. van der Wijngaart, Lab Chip 10, 281 (2010).

${ }^{7}$ R. Bernini, G. Testa, L. Zeni, and P. M. Sarro, Appl. Phys. Lett. 93, 011106 (2008).

${ }^{8}$ G. Testa, Y. Huang, L. Zeni, P. M. Sarro, and R. Bernini, IEEE Photonics Technol. Lett. 22, 616 (2010).

${ }^{9}$ H. K. Chiu, F. L. Hsiao, C. H. Chan, and C. C. Chen, Opt. Express 16, 15069 (2008).

${ }^{10}$ G. Testa, Y. Huang, P. M. Sarro, L. Zeni, and R. Bernini, Opt. Lett. 35, 1584 (2010).

${ }^{11}$ H. Schmidt, D. Yin, J. P. Barber, and A. R. Hawkins, IEEE J. Sel. Top. Quantum Electron. 11, 519 (2005).

${ }^{12}$ F. Vollmer and S. Arnold, Nat. Methods 5, 591 (2008). 